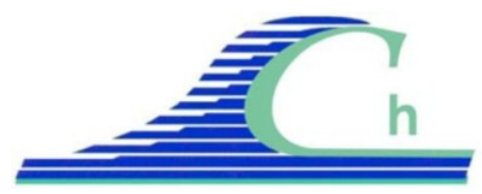

XII ${ }^{\text {ìmes }}$ Journées Nationales Génie Côtier - Génie Civil

Cherbourg, 12-14 juin 2012

DOI:10.5150/jngcgc.2012.111-H C Editions Paralia CFL

disponible en ligne - http://www.paralia.fr - available online

\title{
Caractérisation de sables fins marins de la région de Casablanca (Maroc) en tant que granulats pour béton
}

\section{Sana HICHOUR ${ }^{1}$, Rachid ESSAMOUD ${ }^{1}$, Lhoucine EJJAOUANI ${ }^{2}$}

1. LGSE: Laboratoire de Géoressources sédimentaires et Environnement, Département de Géologie. Faculté des Sciences Ben Msik, B.P. 7955, Casablanca, Maroc.

2. LPEE : Laboratoire Public d'Essais et d'Etudes, 25 rue d'Azilal Casablanca, Maroc. hichoursana@yahoo.fr

\section{Résumé :}

Des alternatives aux exploitations massives du cordon dunaire et au pillage illégal du sable de plage et de dune, ont abouti à la proposition de l'utilisation des sables fins marins (carrières sous-marines) des gisements offshore au Maroc comme solution alternative. Toute valorisation de ces sédiments en tant que granulat, doit satisfaire les normes marocaines relatives aux granulats, mais aussi tenir compte des impacts économiques et environnementaux. Une caractérisation de sables fins marins du point de vue géotechnique et mécanique s'avère nécessaire pour leur classification selon la norme. L'objectif donc est de montrer la conformité de ces sables aux exigences normatives, de lever le doute sur leur utilisation avantageuse au plan économique et de contribuer à une politique environnementale plus équilibrée lorsque leur exploitation est bien contrôlée.

\section{Mots clés :}

Sables fins marins- Granulat - propriété physico-chimiques - Carrières sous-marines Béton - Casablanca - Maroc

\section{Abstract:}

Alternatives to the massive attacks of the offshore bar and looting sand of beach and dune ended to proposition of the use marine fine sands (quarry underwater) of the offshore deposits in Morocco as alternative solution. Any valuation of these sediments as aggregate has to satisfy the Moroccan standards relative to aggregates, but also take into account economic and environmental impacts. A geotechnical and mechanical characterization of marine fine sands is necessary for their classification according to the Moroccan standard NM 10.1.271 (2008). The objective is to take up the conformity of sand in normative requirements, and their advantageous use in the economic plan, which allows our industry to contribute to a more well-balanced environmental politics when their exploitation is controlled.

\section{Keywords:}

Marine fine sands - Aggregates - Physical properties - Chemical properties Underwater quarry - Concrete - Casablanca - Morocco 


\section{Introduction}

L'évolution sans cesse croissante de l'activité de la construction au Maroc a entraîné un essor fulgurant du secteur du Bâtiment et des Travaux Publics -BTP- : Construction de nouvelles villes, de logements économiques, d'autoroutes, de ports, de barrages etc. Ceci a pour conséquence une demande accrue en sable et avec pour corollaire une agression massive du cordon dunaire. De ce fait, le recours à des solutions alternatives à l'utilisation de sable du cordon dunaire, s'avère être indispensable et urgente. Cette étude concerne la valorisation des sables fins marins (carrières sous-marines) des gisements offshore au Maroc. Cette valorisation constitue aujourd'hui un enjeu important.

Cet article traite la caractérisation de ces sables marins en vue de leur valorisation en granulats pour béton (HICHOUR, 2011). Cette phase de caractérisation est indispensable à la description et à la définition de la nature du sédiment : elle doit aussi permettre leur classification selon la norme marocaine NM 10.1.271 (2008).

\section{Matériaux et méthodologie}

\subsection{Présentation du site de prélèvement}

La zone d'étude fait partie de la marge mésétienne, s'étendant depuis Rabat jusqu'à El Jadida (MATHIEU, 1986), dont les fonds rocheux se présentent soit sous forme de prolongement des formations paléozoïques observées sur le continent, soit des formations dunaires quaternaires immergées (DUPLANTIER \& GENSOUS, 1980). Les hauts fonds ne couvrent pas la totalité du secteur. Ils peuvent abriter des cuvettes sédimentaires plus ou moins importantes (ZOURARAH, 2002).

Cette zone, objet de prélèvements d'échantillons couvre une superficie du fond marin de $18,17 \mathrm{~km}^{2}$. Le site forme un quadrilatère dont le grand coté est parallèle à la côte, à une distance d'environ 1,3 km. Elle est localisée à une distance comprise entre 1,3 et $4 \mathrm{~km}$ du littoral actuel qui s'étire en une large côte sableuse de Casablanca à Mohammedia. Ce littoral constitue un ensemble morpho-sédimentaire dont les dynamiques sont largement influencées par les aménagements portuaires des deux villes.

Ce tronçon du littoral est considéré comme globalement sec et ventilé, ce qui le place sous l'influence des actions éoliennes érosives. Mais sa morphologie est davantage dictée par les conditions de houle du large. Celle-ci arrive perpendiculairement à la côte et rencontre à son approche des bancs de sables et arêtes. Ces obstacles favorisent les phénomènes de rectification dans certains secteurs, ce qui participe activement à leur érosion. En revanche, d'autres zones sont en partie abritées par ces houles.

Les installations industrielles et portuaires sur le littoral Casablanca - Mohammedia ont favorisé la capture des matériaux issus de la dérive littorale d’une part, et contribué à la régression de certaines portions de la frange côtière d'autre part, du fait des phénomènes de réfraction générés par les ouvrages (IDRISSI et al., 2004). 


\section{XII ${ }^{\text {èmes }}$ Journées Nationales Génie Côtier - Génie Civil \\ Cherbourg, 12-14 juin 2012}

Les matériaux analysés proviennent des fonds marins localisés entre les isobathes 15 et 27 m (figure 1) Une vingtaine d'échantillons de sable marin a été récolté à l'aide d'une benne Van Veen de façon aléatoire. Leur positionnement a été réalisé selon un GPS à bord d'un navire (Aquastar). Les échantillons de sable ont fait l'objet de plusieurs analyses afin de déterminer leurs caractéristiques géotechniques. L'ensemble des sables bruts ont été prélevés et acheminés au Laboratoire Public d'Essais et d'Etudes en Juillet 2009.

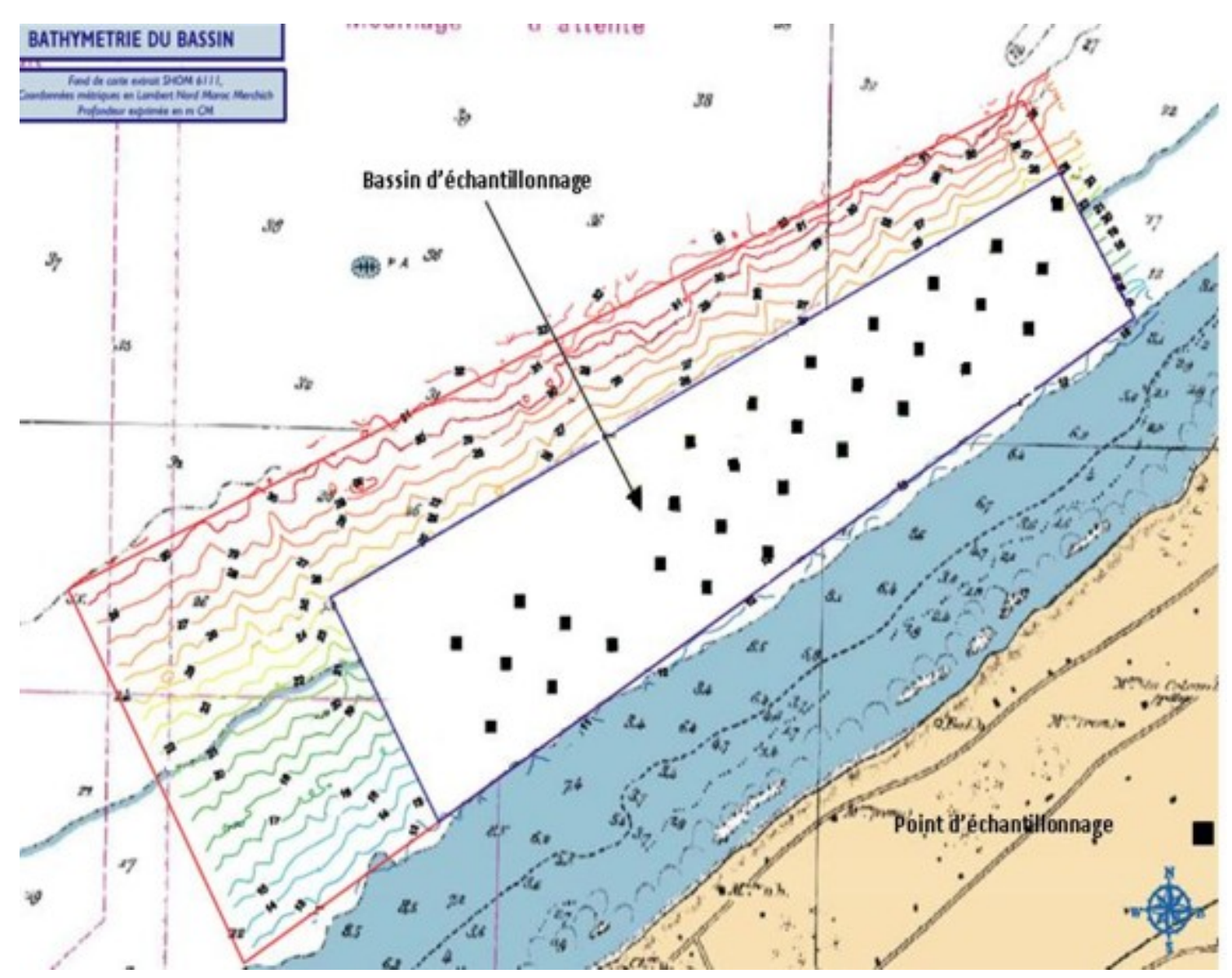

Figure 1.Echantillonnage et points de prélèvements dans la zone d'étude.

\subsection{Méthodologie}

Toute valorisation relative à la réutilisation des sables fins marins en BTP (HICHOUR, 2011 ; MIRAOUI, 2010 ; DUBOIS, 2006 ; SEMCHA, 2006 ; LEVACHER et al., 2011 ; LIMEIRA et al., 2010 ; LIMEIRA et al., 2011 ; LIMEIRA 2011 ; COLIN, 2003) impose une détermination des paramètres géotechniques et chimiques de base des sables fins ainsi que de ses propriétés physiques. Les méthodes de détermination des caractéristiques sont classiques et respectent les normes marocaines en vigueur.

La connaissance des propriétés physico-chimiques de base est nécessaire pour l'identification des caractéristiques géotechniques des sables fins. Elle se fait à partir de 
la détermination de la classe granulaire, de la teneur en fines, du module de finesse (NM00.1.004, 1975), de l'équivalent de sable (NM 10.1.147, 1995), de la valeur au bleu de méthylène (NM 10.1.141, 2008), des teneurs en sulfates (NM 10.1.005, 2008), en chlorures (NM 100.1.140, 1995), du coefficient d'absorption d'eau (NM 10.1.273, 2008) et de la teneur en matière organique (NM 00.1.002, 1975).

\section{Résultats et analyses}

\subsection{Caractérisation géotechnique}

Le sable marin présente une classe granulaire de sable fin à moyen dont les diamètres maximaux varient de 0,25 à $0,315 \mathrm{~mm}$ voir sur la figure 2 . Le module de finesse (Mf) a des valeurs moyennes très faibles pour un usage dans la confection de bétons, elles sont comprises entre 0,8 et 1,5 Mf. Le pourcentage des fines se situe en deçà de $7 \%$, variant de 1,5 à $7 \%$.

L'ensemble des résultats des essais et analyses sont rassemblés dans le tableau 1. On peut observer que les valeurs de l'équivalent de sable ES sont comprises entre $70 \%$ et $90 \%$, les valeurs au bleu du sable, se trouvent entre 0,7 et $1.0 \%$. Les chlorures, les sulfates, les alcalins et l'absorption d'eau présentent des valeurs faibles. Un test colométrique s’est révélé négatif pour la matière organique.

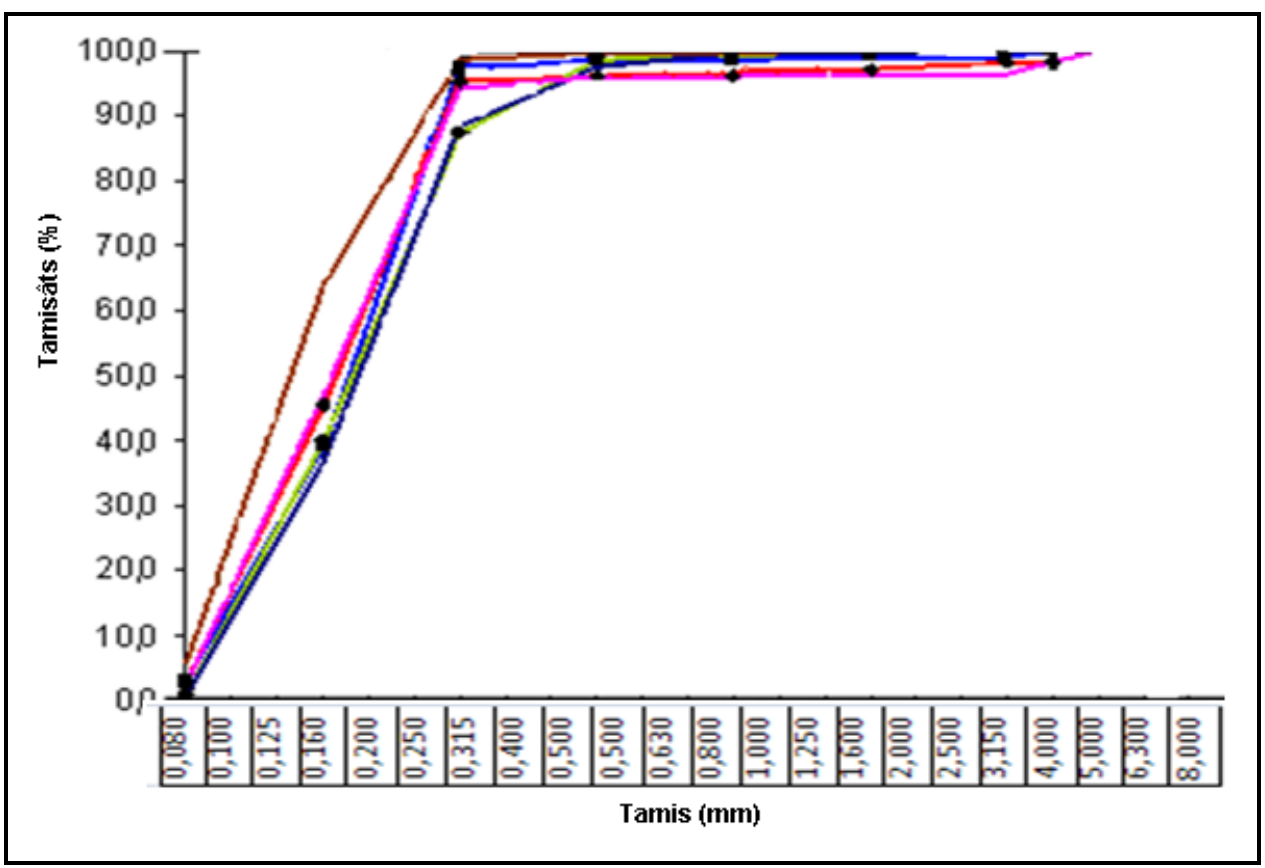

Figure 2. Courbes granulométriques des sables marins étudiés. 


\section{XII ${ }^{\text {èmes }}$ Journées Nationales Génie Côtier - Génie Civil \\ Cherbourg, 12-14 juin 2012}

Tableau 1. Spécifications normatives d'un sable pour béton hydraulique (NM 10.1.271(2008), et caractéristiques des sables marins étudiés.

\begin{tabular}{|c|c|c|c|c|c|c|}
\hline \multirow{2}{*}{ Caractéristiques } & \multirow{2}{*}{$\begin{array}{l}\text { Valeur } \\
\text { spécifiée }\end{array}$} & \multicolumn{4}{|c|}{ Catégorie } & \multirow{3}{*}{$\begin{array}{l}\text { Caractéristique } \\
\text { s géotechniques } \\
\text { des sables } \\
\text { marins }\end{array}$} \\
\hline & & $A$ & B & $C$ & $D$ & \\
\hline \multicolumn{6}{|l|}{ Granularité Gr } & \\
\hline $\begin{array}{l}\text { Passant }(\%) \grave{a} \\
2 D\end{array}$ & $V s i$ & 100 & 100 & 100 & 100 & \multirow{4}{*}{$\begin{array}{l}\text { Sable fin à } \\
\text { moyen }\end{array}$} \\
\hline $\begin{array}{l}\text { Passant }(\%) \grave{a} \\
1,58 \mathrm{D}\end{array}$ & $V s i$ & 99 & 99 & 99 & 99 & \\
\hline \multirow{2}{*}{ Passant (\%) à $D$} & $V s i$ & 85 & 85 & 85 & 85 & \\
\hline & $V S S$ & 99 & 99 & 99 & 99 & \\
\hline \multirow{3}{*}{$\begin{array}{l}\text { Module de finesse } \\
\text { (Mf) }\end{array}$} & $\mathrm{Li}$ & 1,8 & 1.8 & 1.8 & 1.8 & \multirow{3}{*}{$0,8-1,5$} \\
\hline & $L s$ & 3,2 & - & - & - & \\
\hline & $e$ & 0,6 & 0,7 & 0,7 & 0,8 & \\
\hline \multirow{3}{*}{$\begin{array}{l}\text { Teneur en fines } \\
\text { des passants à } \\
0,08 \text { sur fraction } \\
0 / 5 \mathrm{~mm}(\%)\end{array}$} & $L s$ & 12 & 15 & 18 & Pas de & \multirow{3}{*}{$1,5-7$} \\
\hline & e ou & 3 & 5 & 6 & spécificatio & \\
\hline & $C V \leq$ & 20 & 20 & 20 & $\begin{array}{l}n \text { mais FTP } \\
\text { renseignée }\end{array}$ & \\
\hline $\begin{array}{l}\text { Matières } \\
\text { organiques }\end{array}$ & $e$ & \multicolumn{5}{|c|}{ Essai colorimétrique négatif ou essais sur mortiers conformes } \\
\hline
\end{tabular}

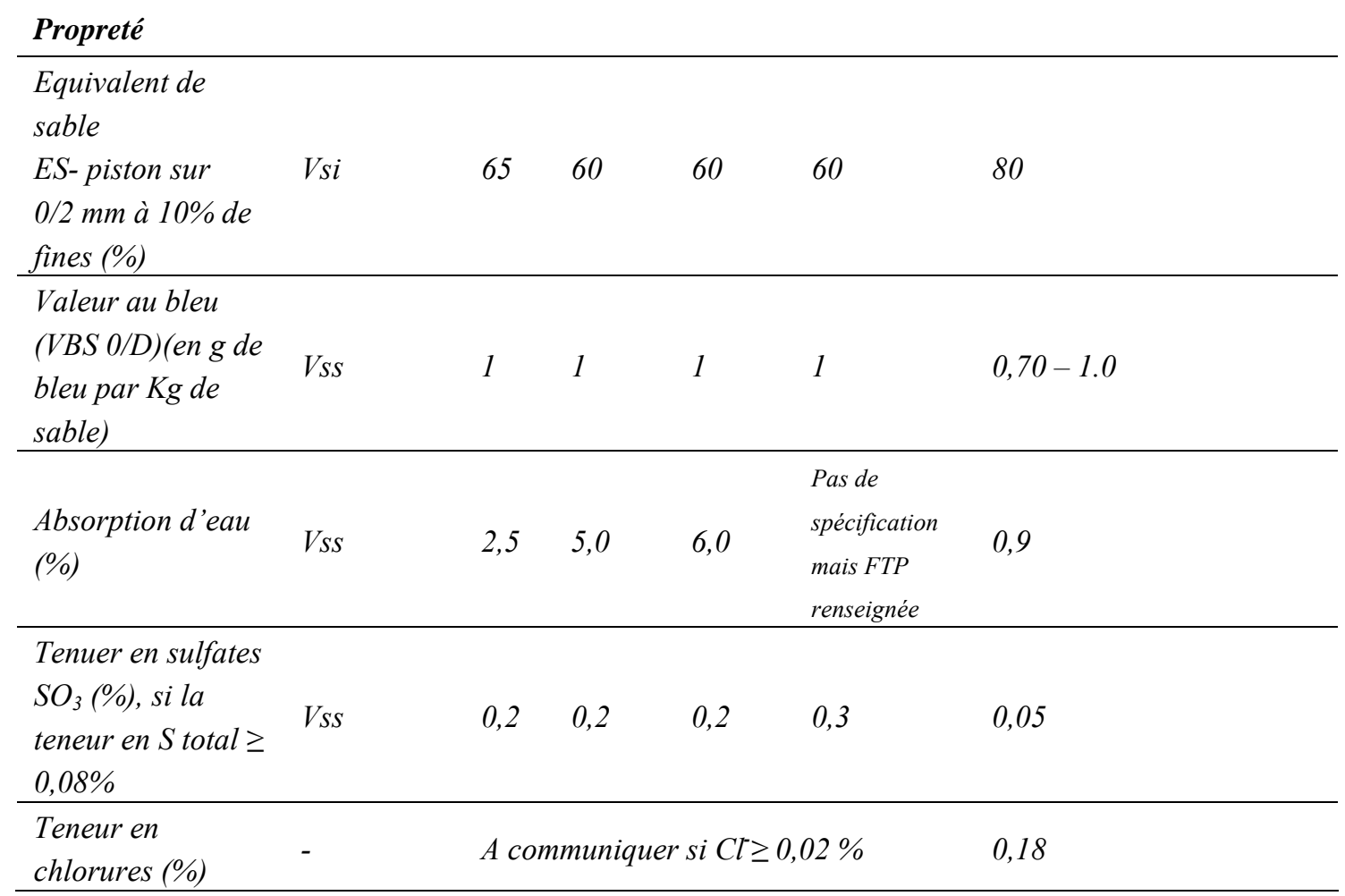

Nota : Li : limite inférieure ; Ls : limite supérieure ; Vsi : valeur spécifique inférieure ; Vss : valeur spécifique supérieure ; $C V$ : coefficient de variation en \% ; FTP : Fiche Technique du Produit 


\subsection{Exigence de la norme marocaine pour les granulats pour bétons}

Les spécifications auxquelles doivent satisfaire les sables sont précisées dans la norme NM 10.1.271 (2008). Cette norme regroupe les caractéristiques que doivent présenter en général les granulats et particulièrement les sables destinés à la confection des bétons hydrauliques, voir le tableau 1.

Les résultats obtenus à partir des essais et analyses d'identification et de caractérisation indiquent que les sables marins, présentent des caractéristiques comparables à celles exigées par la norme pour un béton de catégorie B. La teneur en chlorures reste un peu élevée selon l'exigence de la norme. En outre cette caractéristique doit être renseignée dans la Fiche Technique du Produit - FTP, afin que leur correction soit prise en compte.

\subsection{Teneur en Chlorures Cl-}

La teneur des chlorures reste un peu élevée, sachant bien que cette caractéristique est communiquée systématiquement à l'utilisateur pour permettre l'établissement du bilan des chlorures dans la formulation des bétons.

La teneur moyenne en chlorures des sables marins testés est de 0,18\% (tableau 1). Si l'on considère la proportion de ce sable incorporé dans le mélange le mélange granulaire du béton confectionné qui généralement ne dépasse pas $40 \%$, la teneur en chlorures apportée par ce sable serait inférieure à $0.08 \%$ dans le béton, valeur assez négligeable comparée aux spécifications de la norme NM 10.1.008 (2007) qui est de $0,4 \%$ pour les bétons armés.

\section{Conclusion}

L'analyse des caractéristiques du sable marin a permis de le classer dans la catégorie B selon la norme marocaine relative aux sables utilisés pour la confection des bétons courants de résistance Rc supérieure à $25 \mathrm{MPa}$. La teneur en chlorures apportée par ce sable serait donc inférieure à $0.08 \%$ dans le béton, valeur assez négligeable comparée aux spécifications de la norme NM 10.1.008 (2007).

En définitive, les sables marins constituent l'une des solutions pour satisfaire les besoins présents et futurs qui ne cessent d'augmenter, surtout lorsqu'ils répondent aux exigences environnementales.

\section{Références bibliographiques}

COLIN D. (2003). Valorisation de sédiments fins de dragage en technique routière. Thèse de Doctorat, Université de Caen, 180 p.

DUBOIS V. (2006), Caractérisation physico-mécanique et environnementale des sédiments marins-Application en technique routière, Thèse de doctorat, Ecole des Mines de Douai, $311 \mathrm{p}$. 


\section{XII ${ }^{\text {èmes }}$ Journées Nationales Génie Côtier - Génie Civil \\ Cherbourg, 12-14 juin 2012}

DUPLANTIER F., GENSOUS B. (1980). Etude préliminaire du plateau continental interne de la région de Mohammedia (Maroc). Bull. Inst. Bassin d'Aquitaine, Bordeaux, $n^{\circ}$ 28, pp 81-91.

HICHOUR S. (2011). Les dépôts quaternaires et actuels du littoral et l'offshore de Casablanca-Mohammedia : Etude sédimentologique et géotechnique - Potentialités en géomatériaux et conséquences de l'exploitation sur la stabilité du littoral. Thèse de doctorat, Univ. Hassan II Mohammedia-Casablanca, Faculté des sciences Ben M’Sik, $228 \mathrm{p}$.

IDRISSI M., HOURIMECHE A., REY V., CHAGDALI M. (2004). Impact des installations côtières sur l'évolution du littoral de Mohammedia (Maroc). VIII ${ }^{\text {èmes }}$ Journées Nationales : Génie Côtier - Génie Civil, Compiègne 2004, France, pp 789-796. doi:10.5150/jngcgc.2004.086-I

LEVACHER D., SANCHEZ M., DUAN Z., LIANG Y. (2011). Valorisation en unité pilote de sédiments méditerranéens : étude des caractéristiques géotechniques et de la perméabilité. Revue Paralia, Volume 4, pp 4.1-4.20. doi:10.5150/revue-paralia.2011.004

LIMEIRA DE ARAUJO J. (2011). The beneficial use of dredged marine sand as alternative material for concrete. These de doctorat. Polytechnic University of Catalonia, Barcelone, Spain, 169 p.

MATHIEU R. (1986). Sédiments et foraminifères actuels de la marge continentale du Maroc. Thèse Doctorat d'Etat, Univ. P. M. Curie, Paris, 420 p.

MIRAOUI M. (2010). Mise en oeuvre d'une démarche de prétraitement et de traitement des sédiments de dragage en vue d'une valorisation dans le génie civil. Thèse de Doctorat, Université des sciences et technologies de Lille, 210 p.

SEMCHA A. (2006). Valorisation de sédiments de dragage : Application dans le BTP (cas de barrage algérien). Thèse de doctorat, Université de Reims, 173 p.

ZOURARAH B. (2002). Les processus côtiers actuels et leur impact sur l'environnement littoral des Doukkala, approches hydrodynamique, morphologique et géochimique. Thèse de Doctorat, Univ. Chouaib Dokkali, El Jadida, 228 p.

\section{Normes utilisées}

NM00.1.004 (1975). La détermination de la classe granulaire, la teneur en fines, le module de finesse, $19 \mathrm{p}$.

NM 00.1.002 (1975). La teneur en matière organique, 14 p.

NM 10.1.005 (2008). Les teneurs en sulfates, 120 p.

NM 10.1.008 (2007). Beton : Specifications, performances, production et conformite.

NM 10.1.140(1995). Les teneurs en chlorures, 6 p.

NM 10.1.141(2008). Essais pour déterminer les caractéristiques géométriques des granulats - Qualification des fines - Essai au bleu de méthylène ; (IC 10.1.141) (REV). NM 10.1.147 (1995). Granulats : Equivalent de sable, 9 p.

NM 10.1.271 (2008). Granulats pour bétons hydrauliques (spécifications et conformité). 
Thème 8 - Gestion et valorisation des sédiments marins

NM 10.1.273 (2008). Essais pour déterminer les caractéristiques mécaniques et physiques des granulats - Détermination de la masse volumique réelle et du coefficient d'absorption d'eau. $28 \mathrm{p}$. 